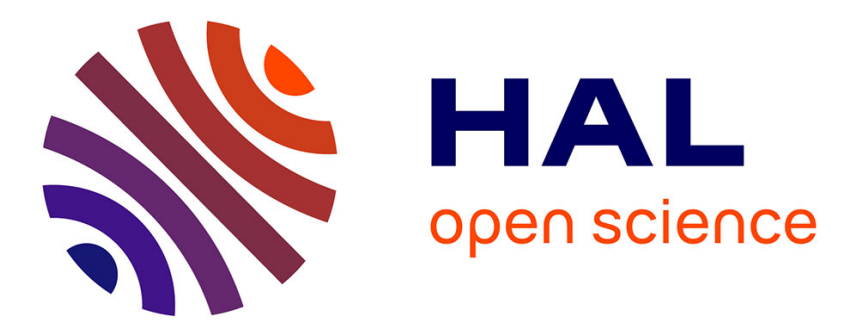

\title{
Détermination du point de fonctionnement d'un capteur solaire plan sans concentration par une méthode graphique
}

\author{
P.-Y. Nizou, A. Chomarat
}

\section{- To cite this version:}

P.-Y. Nizou, A. Chomarat. Détermination du point de fonctionnement d'un capteur solaire plan sans concentration par une méthode graphique. Revue de Physique Appliquée, 1980, 15 (2), pp.143-149. 10.1051/rphysap:01980001502014300 . jpa-00244709

\section{HAL Id: jpa-00244709 https://hal.science/jpa-00244709}

Submitted on 1 Jan 1980

HAL is a multi-disciplinary open access archive for the deposit and dissemination of scientific research documents, whether they are published or not. The documents may come from teaching and research institutions in France or abroad, or from public or private research centers.
L'archive ouverte pluridisciplinaire HAL, est destinée au dépôt et à la diffusion de documents scientifiques de niveau recherche, publiés ou non, émanant des établissements d'enseignement et de recherche français ou étrangers, des laboratoires publics ou privés. 


\title{
Détermination du point de fonctionnement d'un capteur solaire plan sans concentration par une méthode graphique
}

\author{
P.-Y. Nizou \\ Laboratoire de Thermodynamique, U.E.R. de Physique, 2, rue de la Houssinière, 44072 Nantes Cedex, France \\ et A. Chomarat \\ Unité Pédagogique d'Architecture, Avenue Massenet, 44300 Nantes, France
}

(Reçu le 25 juin 1979, révisé le 5 octobre 1979, accepté le 8 octobre 1979)

\begin{abstract}
Résumé. - Les modèles mathématiques traduisant le fonctionnement d'un capteur solaire en régime permanent permettent d'exprimer l'énergie restituée au fluide caloporteur selon une équation caractéristique en fonction de la température moyenne du fluide. Ce rapport présente une méthode simple conduisant à partir de l'équation caractéristique précédente à la construction d'un réseau d'abaques permettant de déterminer le point de fonctionnement d'un capteur solaire plan.

Abstract. - Mathematical models which explain the working of a flat solar collector in a steady state give the rate of useful heat transfered to the working fluid according to a characteristic equation depending on the mean fluid temperature. This paper presents a common method which, from the previous characteristic equation, leads to the drawing of abacuses allowing us to obtain the working point of a flat solar collector.
\end{abstract}

\section{$1^{\text {re }}$ Partie : THÉORIE SIMPLIFIÉE DU CAPTEUR SOLAIRE EN REGIME PERMANENT (RAPPELS)}

1. Généralités. - Un capteur solaire plan ou insolateur à effet de serre peut être le plus souvent représenté par le dispositif schématisé, en coupe, sur la figure 1 .

Un absorbeur dans lequel circule le fluide à réchauffer est exposé sur sa face avant au rayonnement solaire. Afin de réduire les pertes thermiques du dispositif absorbant, la partie avant du capteur proprement dit est constituée d'une couverture transparente au rayonnement solaire, tandis que la partie arrière est constituée d'un matériau isolant. Le rôle de la couverture transparente (en général simple ou double vitrage) est double : elle protège la face absorbante des courants de convection dus au vent et elle récupère par effet de serre une partie du rayonnement infrarouge émis par l'absorbeur.

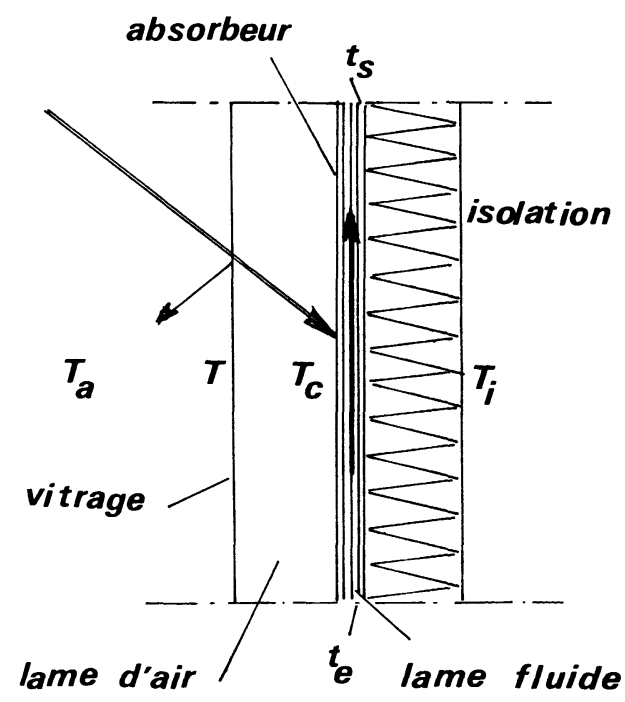

Fig. 1. - Schématisation d'un capteur solaire.

[Diagram of a flat solar collector.] 


\section{Notations utilisées.}

\begin{tabular}{ll} 
Symboles & \multicolumn{1}{c}{ Signification } \\
$t_{\mathrm{e}}$ & température d'entrée de l'air \\
$t_{\mathrm{s}}$ & température de sortie de l'air \\
$\theta$ & température moyenne du fluide $;$ pour u \\
& débit suffisant on a $\theta \simeq \frac{t_{\mathrm{e}}+t_{\mathrm{s}}}{2}$
\end{tabular}

$\Delta \theta \quad$ gain en température entre l'entrée et la sortie $\Delta \theta=t_{\mathrm{s}}-t_{\mathrm{e}}\left[\simeq 2\left(\theta-t_{\mathrm{e}}\right)\right.$ pour un débit suffisant]

$T_{\mathrm{vc}} \quad$ température de la voûte céleste

$T_{\mathrm{a}} \quad$ température ambiante extérieure côté vitrage

$T_{\mathrm{i}} \quad$ température côté isolation ( $T_{\mathrm{i}}$ est la température intérieure d'une habitation si le capteur est intégré à la construction)

$T$ température moyenne de la couverture transparente assimilée ici à un simple vitrage

$T_{\mathrm{c}} \quad$ température moyenne de l'absorbeur

$a_{\mathrm{s}}$ facteur d'absorption du capteur vis-à-vis du rayonnement solaire

$a$ facteur d'absorption du capteur vis-à-vis du rayonnement infrarouge

$\tau_{\mathrm{s}}$ facteur de transmission énergétique du verre vis-à-vis du rayonnement solaire

$\alpha$ facteur d'absorption du verre vis-à-vis du rayonnement infrarouge

$G$ composante suivant la normale au capteur du rayonnement solaire

$\dot{m} \quad$ débit massique du fluide caloporteur, rapporté à l'unité de surface du capteur

$C_{\mathrm{p}} \quad$ capacité thermique massique du fluide caloporteur

$h_{\mathrm{c}} \quad$ coefficient de convection entre absorbeur et fluide caloporteur

$h$ coefficient de convection entre la vitre et l'air extérieur

$h_{\mathrm{rc}} \quad$ coefficient de rayonnement entre l'absorbeur et la vitre

$h_{\text {rvc }}, h_{\text {ra }}$ coefficients de rayonnement entre la vitre et l'atmosphère (rapportés respectivement à la température $T_{\mathrm{vc}}$ et à la température $T_{\mathrm{a}}$ )

$d \quad$ épaisseur de la lame d'air frontale

$\lambda$ conductivité de l'air

$K_{\mathrm{c}} \quad$ conductance globale des échanges thermiques entre absorbeur et simple vitrage

$K \quad$ conductance globale des échanges thermiques entre vitrage et air ambiant

$K_{\mathrm{AR}} \quad$ conductance globale des pertes thermiques de l'absorbeur vers l'arrière

$U$ conductance globale des pertes thermiques du capteur

$E_{\mathrm{r}} \quad$ énergie restituée au fluide par le capteur

$\eta$ rendement global du capteur défini par $\eta=E_{\mathrm{r}} / G$

$F^{\prime} \quad$ facteur caractérisant la qualité du transfert de chaleur entre l'absorbeur et le fluide ce signe placé en exposant d'une température indique que l'on considère l'écart de celle-ci par rapport à la température ambiante $T_{\mathrm{a}}$
K

K

K

sans

dimension

sans

dimension

sans

dimension

sans

dimension

$\mathrm{W} / \mathrm{m}^{2}$

$\mathrm{kg} / \mathrm{s} . \mathrm{m}^{2}$

J/kg K

$\mathrm{W} / \mathrm{m}^{2} \mathrm{~K}$

$\mathrm{W} / \mathrm{m}^{2} \mathrm{~K}$

$\mathrm{W} / \mathrm{m}^{2} \mathrm{~K}$

$\mathrm{W} / \mathrm{m}^{2} \mathrm{~K}$

$m$

$\mathrm{W} / \mathrm{m} \mathrm{K}$

$\mathrm{W} / \mathrm{m}^{2} \mathrm{~K}$

$\mathrm{W} / \mathrm{m}^{2} \mathrm{~K}$

$\mathrm{W} / \mathrm{m}^{2} \mathrm{~K}$

$\mathrm{W} / \mathrm{m}^{2} \mathrm{~K}$

$\mathrm{W} / \mathrm{m}^{2}$

sans

dimension

sans

dimension

par conduction $\left({ }^{1}\right)$ et rayonnement, et est perdu à travers l'isolant de la partie arrière, soit :

$$
\begin{aligned}
a_{\mathrm{s}} \tau_{\mathrm{s}} G=h_{\mathrm{c}}\left(T_{\mathrm{c}}-\theta\right)+\frac{\lambda}{d}\left(T_{\mathrm{c}}-T\right)+ \\
\quad+f(a, \alpha) \sigma\left(T_{\mathrm{c}}^{4}-T^{4}\right)+K_{\mathrm{AR}}\left(T_{\mathrm{c}}-T_{\mathrm{i}}\right)
\end{aligned}
$$

avec

$$
f(a, \alpha)=\left(\frac{1}{a}+\frac{1}{\alpha}-1\right)^{-1}
$$

3.2 EQUILIBRE THERMIQUE DU VITRAGE. - $\mathrm{Au}$ niveau du vitrage, l'énergie reçue de l'absorbeur par conduction et rayonnement est perdue vers l'ambiante par convection et par rayonnement :

$$
\begin{aligned}
\frac{\lambda}{d}\left(T-T_{\mathrm{c}}\right)+f(a, \alpha) \sigma & \left(T^{4}-T_{\mathrm{c}}^{4}\right)= \\
& =h\left(T_{\mathrm{a}}-T\right)+\alpha \sigma\left(T_{\mathrm{vc}}^{4}-T^{4}\right)
\end{aligned}
$$

où $T_{\mathrm{vc}}$ est la température moyenne équivalente de la voûte céleste assimilée à un corps noir $\left({ }^{2}\right)$.

3.3 EQUILIBRE THERMIQUE DU FLUIDE. - L'échange thermique entre l'absorbeur et le fluide s'effectue par convection et conduit à la relation :

$$
\dot{m} C_{\mathrm{p}} \Delta \theta=h_{\mathrm{c}}\left(T_{\mathrm{c}}-\theta\right) .
$$

4. Résolution du système des équations d'équilibre thermique. - Nous nous ramènerons à un système linéaire en introduisant des coefficients d'échange thermique par rayonnement. L'échange par rayonnement entre absorbeur et vitrage s'écrit alors :

$$
f(a, \alpha) . \sigma\left(T_{\mathrm{c}}^{4}-T^{4}\right)=h_{\mathrm{rc}}\left(T_{\mathrm{c}}-T\right)
$$

en posant

$$
h_{\mathrm{rc}}=f(a, \alpha) \cdot \sigma\left(T_{\mathrm{c}}^{2}+T^{2}\right)\left(T_{\mathrm{c}}+T\right) .
$$

( $\left.{ }^{1}\right)$ A partir d'une certaine épaisseur $(d>2,5 \mathrm{~cm})$ les échanges convectifs deviennent prépondérants dans la lame d'air frontale; $\lambda / d$ doit alors être remplacé par un coefficient de convection $h$, traduisant ce phénomène.

$\left({ }^{2}\right)$ N.B. - Diverses formulations ont été proposées pour calculer $T_{\mathrm{vc}}$. Swinbank (cité par Duffie et Beckman [1]) relie cette température à la température ambiante par la relation :

$$
T_{\mathrm{vc}}=0,0552 T_{\mathrm{a}}^{1,5} \text {. }
$$

D'autres auteurs, considérant que cette température équivalente est en fait légèrement inférieure à la température ambiante, sont conduits à poser : $T_{\mathrm{vc}}=T_{\mathrm{a}}-$ Cte (la constante étant comprise entre 7 et $20 \mathrm{~K}$ selon les auteurs). L'une et l'autre de ces formulations ont pu être employées avec un égal succès [2, 3, 4]. Remarquons simplement avec Péri [5] qu'il est difficile de s'accorder sur l'une ou l'autre de ces expressions étant donné l'extrême varia-

bilité de l'état atmosphérique.

LIBRE THERMIQUE DE L'ABSORBEUR. - Au niveau de l'absorbeur à la température $T_{\mathrm{c}}$, l'énergie solaire absorbée $a_{\mathrm{s}} \tau_{\mathrm{s}} G$ est transmise au fluide à la température $\theta$ par convection, au vitrage à la température $T$ 
L'échange par rayonnement avec le ciel s'écrira de même :

$$
\alpha \sigma\left(T_{\mathrm{vc}}^{4}-T^{4}\right)=h_{\mathrm{rvc}}\left(T_{\mathrm{vc}}-T\right)
$$

en posant

$$
h_{\mathrm{rvc}}=\alpha \sigma\left(T_{\mathrm{vc}}^{2}+T^{2}\right)\left(T_{\mathrm{vc}}+T\right) .
$$

Dans ce dernier cas, il sera préférable d'utiliser un coefficient d'échange équivalent relatif à la température ambiante $T_{\mathrm{a}}$; ce coefficient s'écrira :

$$
h_{\mathrm{ra}}=\alpha \sigma\left(T_{\mathrm{vc}}^{2}+T^{2}\right)\left(T_{\mathrm{vc}}+T\right) \frac{T_{\mathrm{vc}}-T}{T_{\mathrm{a}}-T} .
$$

Dans ces conditions, les équations d'équilibre thermique du système deviennent :

$$
\begin{gathered}
a_{\mathrm{s}} \tau_{\mathrm{s}} G=h_{\mathrm{c}}\left(T_{\mathrm{c}}-\theta\right)+K_{\mathrm{c}}\left(T_{\mathrm{c}}-T\right)+K_{\mathrm{AR}}\left(T_{\mathrm{c}}-T_{\mathrm{i}}\right) \\
K_{\mathrm{c}}\left(T-T_{\mathrm{c}}\right)=K\left(T_{\mathrm{a}}-T\right) \\
\eta G=\dot{m} C_{\mathrm{p}} \Delta \theta=h_{\mathrm{c}}\left(T_{\mathrm{c}}-\theta\right)
\end{gathered}
$$

en posant

$$
\begin{aligned}
K_{\mathrm{c}} & =\frac{\lambda}{d}+h_{\mathrm{rc}} \\
K & =h+h_{\mathrm{ra}}
\end{aligned}
$$

Les températures absolues $T, \theta, T_{\mathrm{c}}$ et $T_{\mathrm{i}}$ peuvent s'exprimer en fonction de la température extérieure $T_{\mathrm{a}}$ en posant :

$$
\begin{gathered}
T^{*}=T-T_{\mathrm{a}} ; \quad \theta^{*}=\theta-T_{\mathrm{a}} ; \quad T_{\mathrm{c}}^{*}=T_{\mathrm{c}}-T_{\mathrm{a}} \\
T_{\mathrm{i}}^{*}=T_{\mathrm{i}}-T_{\mathrm{a}} .
\end{gathered}
$$

Avec ces notations, les équations traduisant les équilibres thermiques des différents composants du capteur s'écrivent :

$$
\left.\begin{array}{c}
a_{\mathrm{s}} \tau_{\mathrm{s}} G=h_{\mathrm{c}}\left(T_{\mathrm{c}}^{*}-\theta^{*}\right)+ \\
\quad+K_{\mathrm{c}}\left(T_{\mathrm{c}}^{*}-T^{*}\right)+K_{\mathrm{AR}}\left(T_{\mathrm{c}}^{*}-T_{\mathrm{i}}^{*}\right) \\
K_{\mathrm{c}}\left(T_{\mathrm{c}}^{*}-T^{*}\right)=K T^{*} \\
\eta G=h_{\mathrm{c}}\left(T_{\mathrm{c}}^{*}-\theta^{*}\right)
\end{array}\right\}
$$

$\mathrm{La}$ résolution du système linéaire précédent conduit immédiatement à la relation suivante :

$$
\theta^{*} \text { dét } M=-\left(a_{\mathrm{s}} \tau_{\mathrm{s}} G+K_{\mathrm{AR}} T_{\mathrm{i}}^{*}\right) A+\eta G(A+B)
$$

en posant :

$$
M=\left(\begin{array}{ccc}
h_{\mathrm{c}}+K_{\mathrm{c}}+K_{\mathrm{AR}} & -h_{\mathrm{c}} & -K_{\mathrm{c}} \\
K_{\mathrm{c}} & 0 & -\left(K_{\mathrm{c}}+K\right) \\
h_{\mathrm{c}} & -h_{\mathrm{c}} & 0
\end{array}\right)
$$

matrice des coefficients du système d'équations linéaires (1) (matrice dont le déterminant est :

$$
\text { dét } \left.M=-h_{\mathrm{c}}\left(K K_{\mathrm{c}}+K K_{\mathrm{AR}}+K_{\mathrm{c}} K_{\mathrm{AR}}\right)\right)
$$

et

$$
\begin{aligned}
& A=h_{\mathrm{c}} K+h_{\mathrm{c}} K_{\mathrm{c}} \\
& B=K K_{\mathrm{c}}+K K_{\mathrm{AR}}+K_{\mathrm{c}} K_{\mathrm{AR}} .
\end{aligned}
$$

Dans ces conditions, l'énergie restituée par le capteur s'écrit :

$$
\begin{aligned}
E_{\mathrm{r}}=\eta G=\dot{m} C_{\mathrm{p}} \Delta \theta= & \frac{\text { dét } M}{A+B} \theta^{*}+ \\
& +\frac{A a_{\mathrm{s}} \tau_{\mathrm{s}}}{A+B} G+\frac{A K_{\mathrm{AR}} T_{\mathrm{i}}^{*}}{A+B}
\end{aligned}
$$

relation que l'on peut mettre sous la forme :

$$
\eta G=F^{\prime}\left[a_{\mathrm{s}} \tau_{\mathrm{s}} G-U \theta^{*}+K_{\mathrm{AR}} T_{\mathrm{i}}^{*}\right]
$$

en posant :

$$
F^{\prime}=\frac{A}{A+B}, \quad U=-\frac{\text { dét } M}{A} .
$$

On retrouve ainsi l'équation caractéristique la plus utilisée pour décrire le fonctionnement d'un capteur solaire plan sans concentration. D'un point de vue pratique, il est commode de remplacer $\theta^{*}=\theta-T_{\mathrm{a}}$ par $\theta^{*}=\left(t_{\mathrm{e}}+t_{\mathrm{s}}\right) / 2-T_{\mathrm{a}}$, approximation tout à fait justifiée si le débit est suffisant. Sacadura [6] montre que cette approximation permet d'atteindre la valeur exacte de $\eta G$ à moins de $2 \%$ près dès que $\dot{m} C_{\mathrm{p}} / U$ est supérieur à 2 .

- $F^{\prime}$ est un nombre sans dimension qui caractérise la qualité du transfert de chaleur entre fluide et absorbeur. Il dépend un peu des conditions de fonctionnement (puisqu'il contient par exemple $h_{\mathrm{c}}$ qui est fonction du débit) mais il est surtout déterminé par les caractéristiques de construction de l'absorbeur. $F^{\prime}$ est par définition inférieur à 1 . Pour un absorbeur de bonne qualité $F^{\prime}$ peut atteindre des valeurs supérieures à 0,9 .

- Les grandeurs $U$ et $K_{\mathrm{AR}}$ (nécessairement positives) apparaissent comme des conductances thermiques ou coefficients d'échange. $U$ représente la conductance globale des pertes thermiques du capteur. $K_{\mathrm{AR}}$ représente la conductance des pertes thermiques du capteur vers l'arrière. $\mathrm{Si}$ on désigne par $K_{\mathrm{Av}}$ la conductance globale des pertes thermiques du capteur vers l'avant, on a évidemment : $U=K_{\mathrm{Av}}+K_{\mathrm{AR}}$. En toute rigueur $U$ n'est pas une constante mais on peut en prendre une valeur moyenne sur l'ensemble de la plage de fonctionnement du capteur.

L'équation caractéristique (3) est tout à fait générale et peut être employée quel que soit le type de capteur considéré : capteur à eau ou à air, couverture à simple ou double vitrage, absorbeur à plaque ou à tubes soudés, etc. Les ouvrages spécialisés [1,7], donnent la valeur des paramètres $F^{\prime}, U, K_{\mathrm{AR}}$ pour les configurations les plus utilisées.

Des valeurs moyennes ont été proposées pour représenter les coefficients caractéristiques des capteurs à eau les plus courants. Nous en donnons ici 
deux exemples définis pour des données climatiques moyennes avec $T_{i}^{*}=0$ :

- capteurs plans à une vitre

$\eta=0,8-7,5 \frac{\theta^{*}}{G} \quad$ d'après la référence $[8]$

$\eta=0,75-7 \frac{\theta^{*}}{G} \quad$ d'après les références $[2,9]$

- capteurs plans à double vitrage

$\eta=0,6-4 \frac{\theta^{*}}{\bar{G}} \quad$ d'après la référence $[8]$

$\eta=0,65-\frac{\theta^{*}}{G} \quad$ d'après les références $[2,9]$.

Remarque. - Dans la configuration particulière proposée par la figure 1 (cas le plus courant) et qui nous a conduit à l'éq. (2), on remarque que l'on a :

\section{$2^{\text {e }}$ Partie : REPRÉSENTATION GRAPHIQUE DE L'ÉQUATION CARACTÉRISTIQUE (3)}

1. Energie restituée au fluide par le capteur. Réseau iso-ensoleillement. - 1.1 CETTE ÉNERGIE A POUR VALEUR :

$$
\begin{aligned}
E_{\mathrm{r}}=\eta G=\dot{m} C_{\mathrm{p}} \Delta \theta=- & F^{\prime} U \theta^{*}+ \\
& +F^{\prime} a_{\mathrm{s}} \tau_{\mathrm{s}} G+F^{\prime} K_{\mathrm{AR}} T_{\mathrm{i}}^{*} .
\end{aligned}
$$

Pour une énergie reçue $G$ constante, l'énergie restituée s'exprime ainsi linéairement en fonction de la température $\theta^{*}$ du fluide en circulation. Dans un système d'axes $E_{\mathrm{r}}, \theta^{*}$ les droites obtenues en donnant à $G$ des valeurs constantes auront l'allure de celle figurée ci-contre (Fig. 2).

1.2 RÉSEAU ISO-ENSOLEILLEMENT. - 1.2.1 On donne à $G$ des valeurs définies par $G=n \times$ Cte avec $n=1,2,3, \ldots, n$. - Les points d'intersection avec les axes sont définis par :

$$
\begin{array}{ll}
\theta^{*}=0 & E_{\mathrm{r}}=F^{\prime} a_{\mathrm{s}} \tau_{\mathrm{s}} G+F^{\prime} K_{\mathrm{AR}} T_{\mathrm{i}}^{*} \\
E_{\mathrm{r}}=0 & \theta^{*}=\frac{a_{\mathrm{s}} \tau_{\mathrm{s}}}{U} G+\frac{K_{\mathrm{AR}}}{U} T_{\mathrm{i}}^{*} .
\end{array}
$$

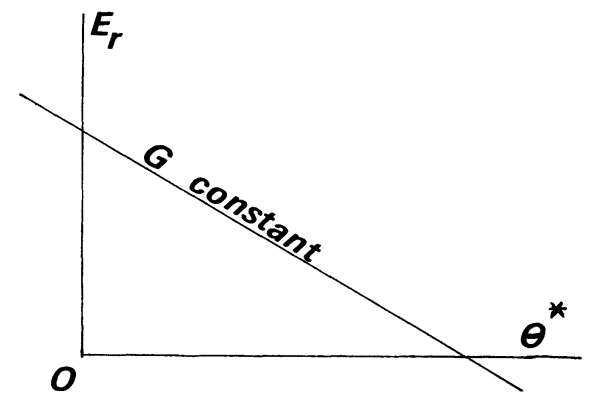

Fig. 2. - Energie restituée par un capteur en fonction de l'écart de température $\theta^{*}=\theta-T_{\mathrm{a}}$ pour un ensoleillement $G$ constant.

[Rate of useful heat transfered to the working fluid versus $\theta^{*}=\theta-T_{\mathrm{a}}$, for a given solar radiation $G$.]

Ces points sont équidistants si on donne à $G$ les valeurs définies au début de ce paragraphe.

1.2.2 Droites particulières du réseau iso-ensoleillement. - 1.2.2.1 $G=0 .-$ En reportant cette valeur particulière de $G$ dans (4) il vient :

$$
\begin{array}{ll}
\theta^{*}=0 & E_{\mathrm{r}}=F^{\prime} K_{\mathrm{AR}} T_{\mathrm{i}}^{*} \\
E_{\mathrm{r}}=0 & \theta^{*}=\frac{K_{\mathrm{AR}}}{U} T_{\mathrm{i}}^{*}
\end{array}
$$

Suivant la valeur relative de la température intérieure par rapport à la température extérieure on obtient l'un ou l'autre des graphes de la figure 3.

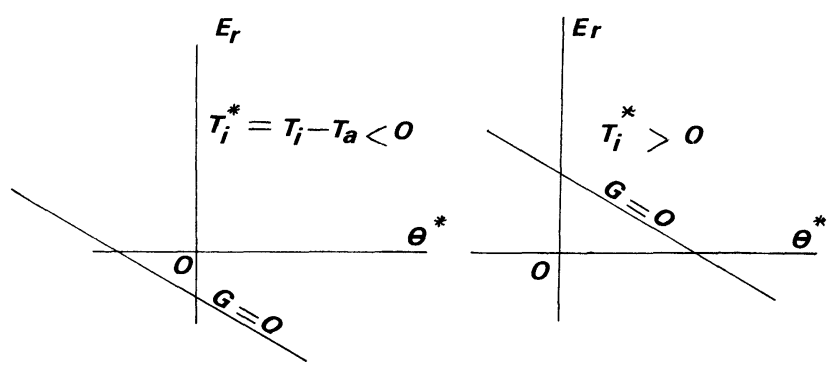

Fig. 3. - Positions possibles de la droite correspondant à l'ensoleillement nul.

[Possible positions of the straight line corresponding to a zero solar radiation.]

1.2.2.2 Seuil de fonctionnement $\left(E_{\mathrm{r}}>0\right)$. - Le seuil de fonctionnement correspond à la valeur minimale de $G$ que l'on désigne par $\hat{G}$ telle que la droite du réseau correspondant à $\hat{G}$ passe par 0 (Fig. 4). Cette valeur particulière de $G$ est obtenue à partir de l'équation (3) en prenant :

ainsi

$$
E_{\mathrm{r}}=\theta^{*}=0
$$

$$
\hat{G}=\frac{-K_{\mathrm{AR}} T_{\mathrm{i}}^{*}}{a_{\mathrm{s}} \tau_{\mathrm{s}}} .
$$




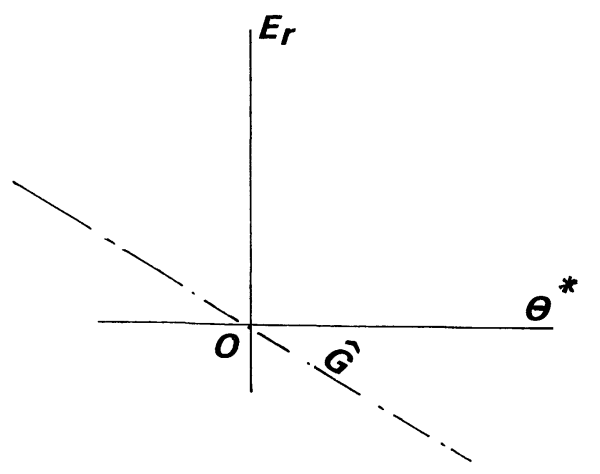

Fig. 4. - Seuil de fonctionnement.

[Working threshold.]

En résumé :

- si $T_{\mathrm{i}}^{*}<0$ le capteur fonctionnera $\left(E_{\mathrm{r}}>0\right)$ pour une énergie reçue $G>\hat{G}$,

- si $T_{\mathrm{i}}^{*} \geqslant 0$ (cas habituel) le seuil de fonctionnement est défini par $G>0$.

2. Rendement du capteur. Réseau iso-rendement. Désignons par $\hat{\eta}$ une valeur particulière du rendement $\eta$. Quelle que soit $\hat{\eta}$, l'éq. (3) devient :

soit

$$
\hat{\eta} G=F^{\prime}\left[a_{\mathrm{s}} \tau_{\mathrm{s}} G-U \theta^{*}+K_{\mathrm{AR}} T_{\mathrm{i}}^{*}\right]
$$

$$
G=\frac{F^{\prime}}{\hat{\eta}-F^{\prime} a_{\mathrm{s}} \tau_{\mathrm{s}}}\left[K_{\mathrm{AR}} T_{\mathrm{i}}^{*}-U \theta^{*}\right] .
$$

En reportant cette valeur de $G$ dans l'équation générale (3) :

$$
E_{\mathrm{r}}=\frac{-F^{\prime} U \hat{\eta}}{\hat{\eta}-F^{\prime} a_{\mathrm{s}} \tau_{\mathrm{s}}} \theta^{*}+\frac{F^{\prime} K_{\mathrm{AR}} \hat{\eta}}{\hat{\eta}-F^{\prime} a_{\mathrm{s}} \tau_{\mathrm{s}}} T_{\mathrm{i}}^{*} .
$$

Dans le même système d'axes que précédemment $\left[\mathrm{E}_{\mathrm{R}}, \theta^{*}\right]$ les points représentatifs correspondant à un rendement constant $\hat{\eta}$ sont situés sur une droite dont l'équation est donnée en (5).

2.1 Point Particulier. - Pour $E_{\mathrm{r}}=0$, on a

$$
\theta^{*}=\frac{K_{\mathrm{AR}} T_{\mathrm{i}}^{*}}{U},
$$

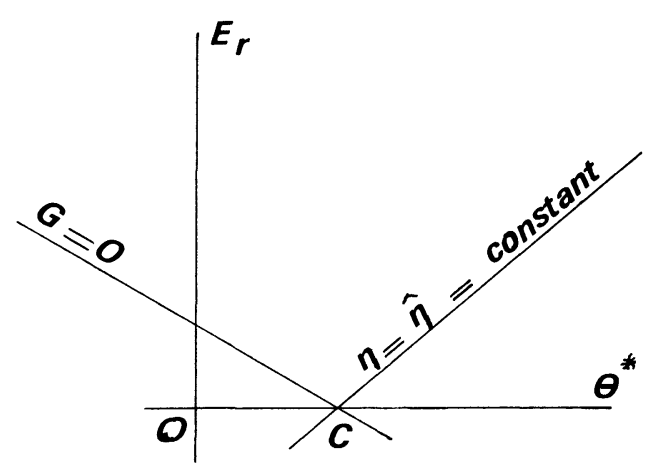

Fig. 5. - Point de concours des droites iso-rendement.

[Intersection of the iso-efficiency straight lines.] valeur indépendante de $\hat{\eta}$. Ainsi, toutes les droites iso-rendement passent par le point $C\left[0, \frac{K_{\mathrm{AR}}}{U} T_{\mathrm{i}}^{*}\right]$ qui a été précédemment déterminé $(1.2 .2 .1)$ comme étant le point où la droite $G=0$ coupe l'axe des $\theta^{*}$ (Fig. 5). Ce point est très voisin de l'origine pour tous les capteurs correctement isolés à l'arrière.

2.2 COEFFICIENT ANGULAIRE DES DROITES ISO-RENDEMENT. - $\bullet$ Pour $\hat{\eta}<F^{\prime} a_{\mathrm{s}} \tau_{\mathrm{s}}$ le coefficient angulaire est positif.

- Pour $\hat{\eta}=F^{\prime} a_{\mathrm{s}} \tau_{\mathrm{s}}$ le coefficient angulaire est infini.

- $\hat{\eta}=F^{\prime} a_{\mathrm{s}} \tau_{\mathrm{s}}$ correspond au rendement maximum théorique du capteur. Cette valeur ne peut donc pas être dépassée. Les deux réseaux iso-ensoleillement et iso-rendement regroupés sur le même système d'axes $E_{\mathrm{r}}, \theta^{*}$ permettent de déterminer le fonctionnement du capteur et sa régulation en fonction des données d'entrée (Fig. 6).

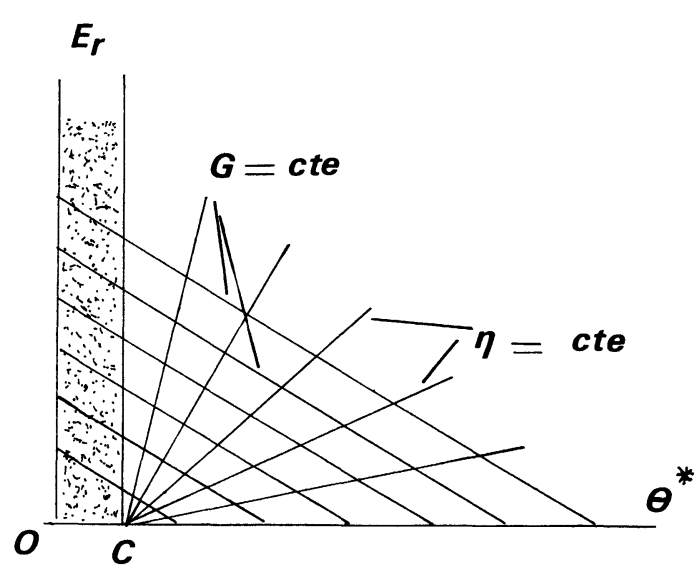

Fig. 6. - Allure générale des réseaux iso- $G$ et iso- $\eta$ : les points de fonctionnement possibles se situent hors de la zone ombrée.

[General appearance of network : the possible working points are situated outside of the shaded area.]

3. Exemples d'application des abaques. - 3.1 VALEURS NUMÉRIQUES PERMETTANT DE DÉFINIR LES RÉSEAUX. - On considère, pour cet exemple, un capteur à air, à simple vitrage, placé à l'extérieur, dont les caractéristiques sont :

$$
\begin{aligned}
& a_{\mathrm{s}} \tau_{\mathrm{s}}=0,72 \quad \text { [facteur optique du capteur] } \\
& F^{\prime}=0,81 \quad \text { valeurs moyennes sur l'ensem- } \\
& \left.U=7,2 \mathrm{~W} / \mathrm{m}^{2} \mathrm{~K}\right\} \text { ble de la plage de fonctionne- } \\
& T_{\mathrm{i}}^{*}=0 \quad \text { ment du capteur }\left({ }^{2}\right) \text {. }
\end{aligned}
$$

${ }^{2}$ ) Les produits $F^{\prime} a_{\mathrm{s}} \tau_{\mathrm{s}}$ et $F^{\prime} U$ (dont la connaissance numérique suffit à définir les réseaux) sont normalement accessibles à partir des données du constructeur si celles-ci sont présentées selon la norme expérimentale P 50-501 [9]. 
Remarque : On démontre sans difficulté que les droites du réseau iso-rendement, obtenues en donnant à $\eta$ des valeurs en progression arithmétique, coupent chaque droite du réseau iso-ensoleillement suivant des segments de longueurs égales. Cette remarque facilite la construction du réseau iso-rendement qui est ainsi obtenue à partir des valeurs $\eta=0$ sur l'axe $\theta^{*}$ et $\eta=F^{\prime} a_{\mathrm{s}} \tau_{\mathrm{s}}$ sur l'axe $E_{\mathrm{r}}$.

Les réseaux iso-ensoleillement et iso-rendement correspondant aux valeurs numériques ci-dessus sont représentés figure 7 .

3.2 Utilisation Des RÉSEAUX. - 3.2.1 Détermination d'un point de fonctionnement. - On se propose de déterminer, pour une valeur de l'ensoleillement $G$ donnée, le point de fonctionnement du capteur correspondant à une température moyenne $\theta$ du fluide, les températures ambiante et d'entrée étant également supposées connues :

\section{Exemple :}

- Ensoleillement $\quad G=800 \mathrm{~W} / \mathrm{m}^{2}$.

- Température ambiante $T_{\mathrm{a}}=0{ }^{\circ} \mathrm{C}$.

- Température d'entrée $t_{\mathrm{e}}=18^{\circ} \mathrm{C}$.

- Température du fluide $\theta=30^{\circ} \mathrm{C}$.

Des relations définies dans les paragraphes précédents, on peut déduire :

$$
\begin{aligned}
\theta^{*} & =\theta-T_{\mathrm{a}}=30^{\circ} \mathrm{C} \\
\Delta \theta & =2\left(\theta-t_{\mathrm{e}}\right)=24^{\circ} \mathrm{C}
\end{aligned}
$$

$G$ et $\theta^{*}$ définissent alors sur le graphe précédent le point de fonctionnement $P$ permettant de déterminer la valeur du rendement $\eta$ et le débit du fluide $\dot{m} C_{\mathrm{p}}$ à assurer :

$$
\begin{aligned}
\eta & \simeq 0,36 \\
\dot{m} C_{\mathrm{p}} \Delta \theta & \simeq 290 \mathrm{~W} / \mathrm{m}^{2}
\end{aligned}
$$$$
\text { soit : } \quad \dot{m} C_{\mathrm{p}} \simeq 12 \mathrm{~W} / \mathrm{m}^{2} \mathrm{~K} \text {. }
$$

On note par ailleurs sur le réseau iso-ensoleillement la valeur du rayonnement de seuil $G=300 \mathrm{~W} / \mathrm{m}^{2}$ correspondant au rayonnement minimum nécessaire, compatible avec les conditions de température imposées.

3.2.2 Déplacement du point de fonctionnement pour une régulation donnée. - 3.2.2.1 Fonctionnement à débit constant. - L'énergie restituée au fluide par le capteur a pour valeur :

$$
E_{\mathrm{r}}=\dot{m} C_{\mathrm{p}} \Delta \theta
$$

avec $\dot{m} C_{\mathrm{p}}=$ débit du fluide (par unité de surface du capteur) et

$\Delta \theta=\theta-t_{\mathrm{e}}=\left(\theta-T_{\mathrm{a}}\right)+\left(T_{\mathrm{a}}-t_{\mathrm{e}}\right)=\theta^{*}+\left(T_{\mathrm{a}}-t_{\mathrm{e}}\right)$.

Dans le cas d'un fonctionnement régulé à débit constant, on voit que l'énergie restituée $E_{\mathrm{r}}$ s'exprime sous une forme linéaire en fonction de $\theta^{*}$ :

$$
E_{\mathrm{r}}=\text { Cte } \times\left[\theta^{*}+T_{\mathrm{a}}-t_{\mathrm{e}}\right] .
$$

Les points de fonctionnement sont situés sur des droites. Dans le cas particulier où la température

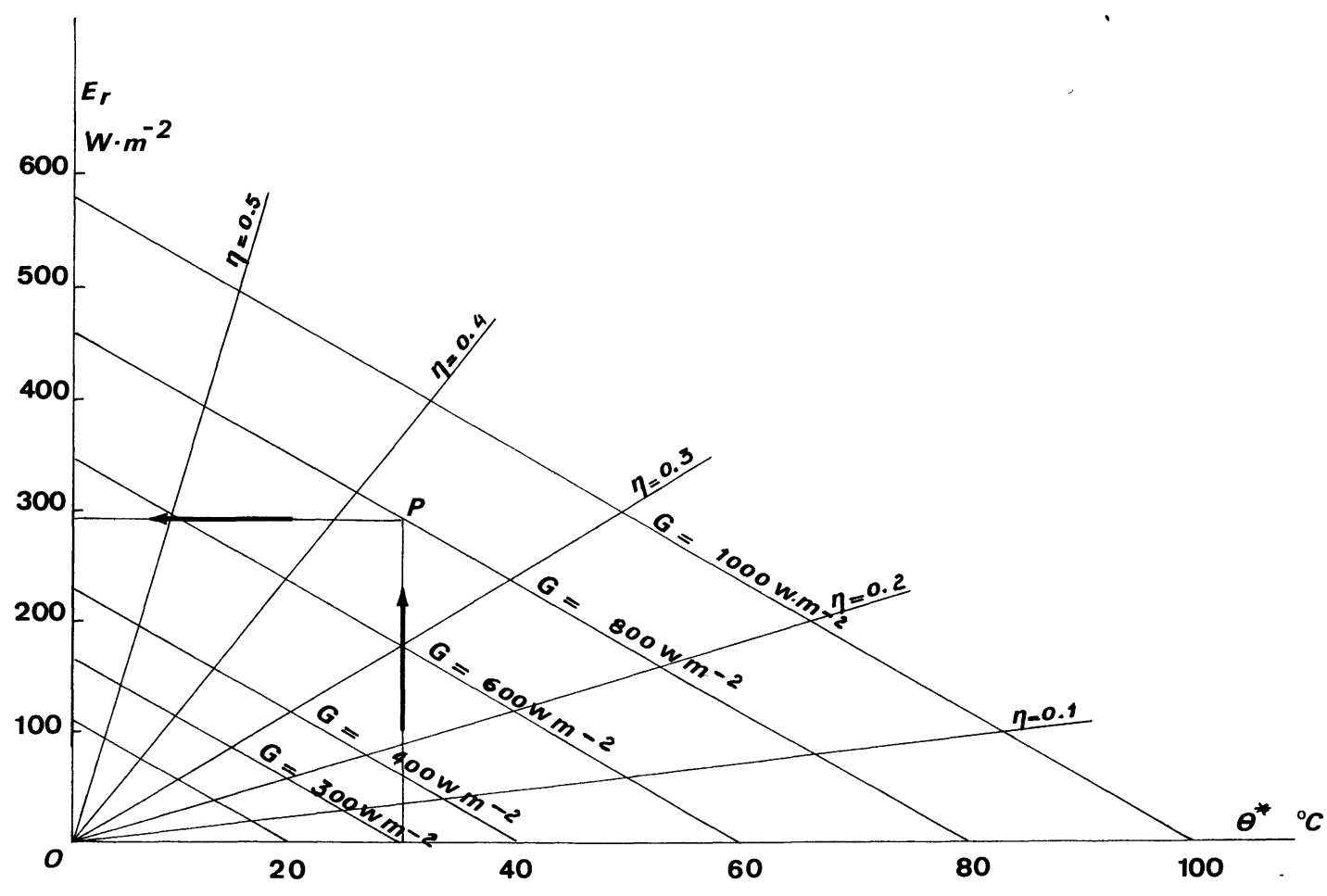

Fig. 7. - Réseau correspondant à un capteur de caractéristiques : $a_{\mathrm{s}} \tau_{\mathrm{s}}=0,72 ; F^{\prime}=0,81 ; U=7,2 \mathrm{~W} / \mathrm{m}^{2} \mathrm{~K} ; T_{\mathrm{i}}^{*}=0$.

[Network for a given flat solar collector.] 
ambiante $T_{\mathrm{a}}$ est égale à la température d'entrée $t_{\mathrm{e}}$, ces droites passent par l'origine et sont donc confondues avec celles du réseau iso-rendement. Le fonctionnement à débit constant correspond ainsi, dans ce cas, à un fonctionnement à rendement constant.

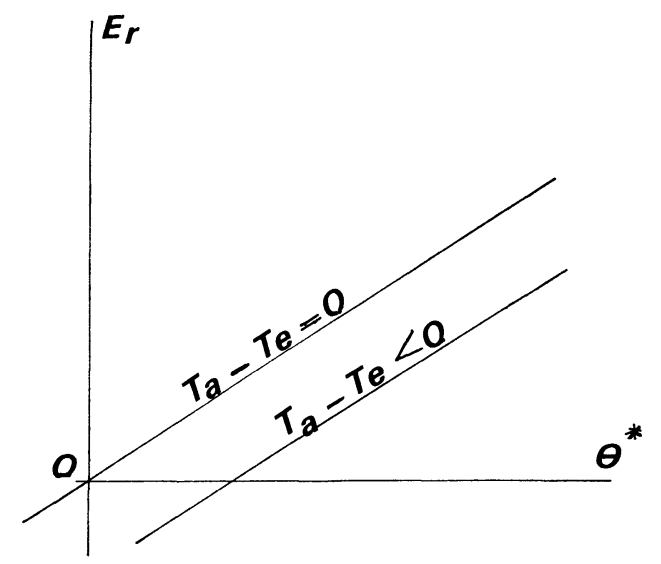

Fig. 8. - Régulation à débit constant.

[Constant flow regulation.]

3.2.2.2 Fonctionnement à différence de tempérarature $\theta^{*}$ constante. $-\mathrm{Si}$ la différence de température $\theta^{*}=\theta-T_{\mathrm{a}}$ est maintenue constante, on remarque que le débit doit varier dans le même sens que l'ensoleillement.

Sur le graphe précédent, les points représentant l'état d'équilibre sont situés sur une verticale correspondant à l'équation $\theta^{*}=$ Cte.

On peut noter que le rendement s'améliore lorsque l'ensoleillement augmente.

3.2.2.3 Fonctionnement à température $\theta$ constante. - On suppose que la' température du fluide $\theta$ est maintenue constante. Si on admet par hypothèse que la température extérieure $T_{\mathrm{a}}$ varie, sinon proportionnellement, du moins dans le même sens que l'énergie solaire, l'écart entre la température $\theta$ et la température ambiante, c'est-à-dire $\theta^{*}$, diminue lorsque l'énergie due au rayonnement solaire augmente.

Contrairement au cas précédent, les points représentatifs des états d'équilibre sont situés sur une courbe qui s'incurve vers l'axe vertical, donc vers les rendements importants.

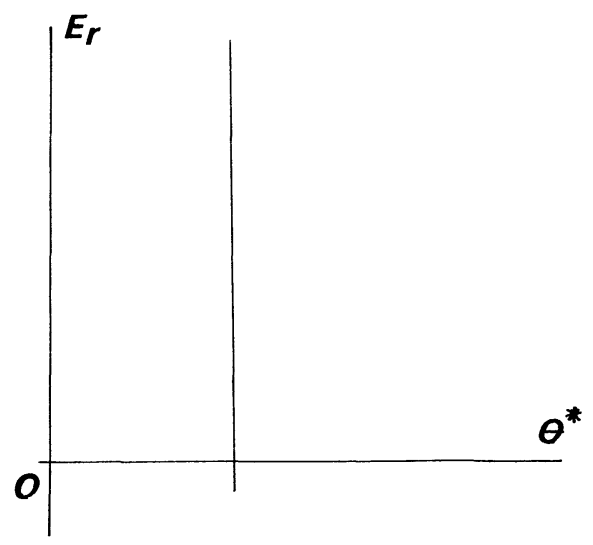

Fig. 9. - Régulation à différence de température $\theta^{*}$ constante. [Regulation with a constant temperature difference $\theta^{*}$.]

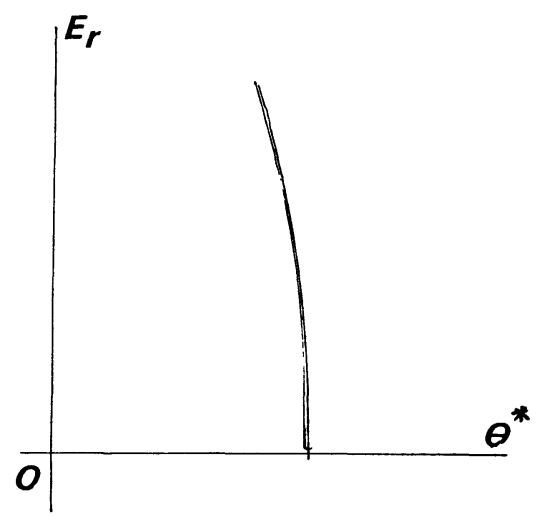

Fig. 10. - Régulation à température $\theta$ constante.

[Regulation with a constant temperature $\theta$.]

4. Conclusion. - Il est clair que les abaques obtenus permettent d'établir le point de fonctionnement du capteur quels que soient les paramètres d'entrée $(\theta, G, \dot{m}, \ldots)$ soit d'une manière directe comme dans l'exemple précédent, soit par approximations successives dans certains cas.

Ce mode de représentation, qui permet de visualiser d'une façon simple le domaine de fonctionnement d'un capteur solaire, peut ainsi aider à la recherche de la définition optimale d'un système de captation (choix du type de capteur, choix de la régulation...) compte tenu des contraintes d'utilisation imposées.

\section{Bibliographie}

[1] Duffie, J. A., Beckman, W. A., Solar Energy Thermal Processes (John Wiley) 1974.

[2] Berhondo, P., Etude théorique complémentaire des capteurs plans solaires à eau. Vérification expérimentale. Rapport HC 312 W 833, 1975, EDF Centre des Renardières.

[3] RoCaries, F., Simulation informatique et habitat solaire. Cah. Tech. Moniteur 19 (1978) 83-89.

[4] Flechon, J., Température d'équilibre d'un collecteur plan dans des conditions réelles définies, Revue Phys. Appl. 11 (1976) $743-750$.

[5] PerI, G., Les capteurs convertisseurs héliothermiques sans concentration. Cahiers A.F.E.D.E.S. no 4, déc. (1977).
[6] Sacadura, J. F. O., Equations caractéristiques des capteurs solaires plans sans concentration. Rev. Gen. Therm., $\mathrm{n}^{\circ} 171$ (1976) 217-223.

[7] Utilisation de l'énergie solaire dans l'habitat. Cycle d'enseignement supérieur, CAST-IFCE-AFEDES, 1977.

[8] GICQUEL, R., Méthode pour évaluer l'énergie solaire fournie par un insolateur plan. Rev. Gén. Therm., no 164-165 (1975) 581-589.

[9] Norme expérimentale P. 50-501. Capteurs solaires : Mesure des performances thermiques. AFNOR Déc. 1977. 